\title{
Wave dynamic processes induced by a supersonic projectile discharging from a shock tube
}

\author{
Zonglin Jianga) \\ Key Laboratory of High Temperature Gas Dynamics, Institute of Mechanics, Chinese Academy of Sciences, \\ 15 Zhong Guan Cun Road, Beijing 100080, People's Republic of China
}

(Received 29 May 2002; accepted 31 January 2003; published 5 May 2003)

\begin{abstract}
A numerical study on wave dynamic processes occurring in muzzle blast flows, which are created by a supersonic projectile released from the open-end of a shock tube into ambient air, is described in this paper. The Euler equations, assuming axisymmetric flows, are solved by using a dispersion-controlled scheme implemented with moving boundary conditions. Three test cases are simulated for examining friction effects on the muzzle flow. From numerical simulations, the wave dynamic processes, including two blast waves, two jet flows, the bow shock wave and their interactions in the muzzle blasts, are demonstrated and discussed in detail. The study shows that the major wave dynamic processes developing in the muzzle flow remain similar when the friction varies, but some wave processes, such as shock-shock interactions, shock-jet interactions and the contact surface instability, get more intensive, which result in more complex muzzle blast flows.

(C) 2003 American Institute of Physics. [DOI: 10.1063/1.1566752]
\end{abstract}

\section{INTRODUCTION}

The muzzle blast flow has been an interesting topic for a century since it is closely related with the weapon firing problem, one aspect of which is the potential reduction of the weapon blast noise resulting in hearing impairment of people in the vicinity. ${ }^{1-5}$ Recently, it has gained more attention from the RAM accelerator research, ${ }^{6}$ where the shock wave interaction between a projectile and its launch tube is emphasized, and the ballistic range test ${ }^{7}$ for re-entry physics, in which the real gas effect induced by the bow shock in front of the projectile is investigated. Wave dynamic processes occurring in the muzzle blast flow are schematically shown in Fig. 1 as a shock-tube/projectile problem and described briefly as follows. Assuming that a shock tube has an open end to ambient air, in which a projectile moves at a supersonic speed, a precursor shock wave driven by the projectile propagates in the shock tube and ahead of the projectile which acts like a piston. The pressure is higher behind the projectile and lower in front of it due to the friction force between the projectile and the shock tube wall, which maintains a balance between the driving and the drag forces acting on the projectile. When the precursor shock wave discharges from the open end, the first blast develops, which results in shock-wave diffraction with an associated starting vortex ring and a jet flow. Later, the projectile itself moves out of the shock tube and interacts with the diffracting shock system and the jet flow. Meanwhile, the high pressure gas (the propellant gas) behind the projectile expands out of the shock tube and the second blast develops. The second blast can overtake the projectile and the first blast, but these two blasts will be overtaken again later by the projectile. So, this flow field is characterized by two blast waves, two jet flows,

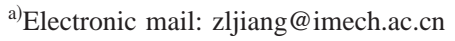

and a bow shock wave. Therefore, it is more interest to investigate from the viewpoint of aerodynamics than just from the weapon blast noise reduction.

The wave dynamic phenomena were reviewed by Glass ${ }^{2}$ from the viewpoint of aerodynamics by presenting a series of photographs showing the emergence of a 0.3 -in.-diam bullet from a rifle at a Mach number of 2.0. The interaction between the propellant gas blast and the first blast was described. Detailed visualization of muzzle blasts was reported by Schmidt et $a .^{3}$ by using a time-resolved, spark shadowgraph technique. The strong coupling between the two blasts was observed, but their interaction was not clearly observable due to the propellant gas being full of dust and smoke. More work ${ }^{1,5}$ was devoted to modeling of blast wave physics in the region far from the jet flow in terms of wellestablished theories for spherical blast waves. The work is helpful for sonic boom reduction in military application, but not very useful for understanding wave dynamic processes in the muzzle blasts. Considering that the muzzle blasts are usually characterized by two blast waves, two jet flows, and the shock-wave/moving-body interaction, Jiang et al. ${ }^{8}$ conducted a numerical study on the muzzle blast by modeling it as a shock-tube/projectile problem. The detailed observation on the wave dynamic processes occurring in the vicinity of the muzzle and around the projectile was reported. From their work, it was found that these wave processes are closely coupled together, and can be neither clearly visualized experimentally because of dusty propellant gases, nor modeled with classic blast theory because of nonlinearity of the wave processes due to complex interactions of various wave phenomena. However, the friction between the projectile and the tube wall was neglected in their work, which results in the second blast being much weaker than in the real physical case.

Aiming at more realistic test cases, the present study 


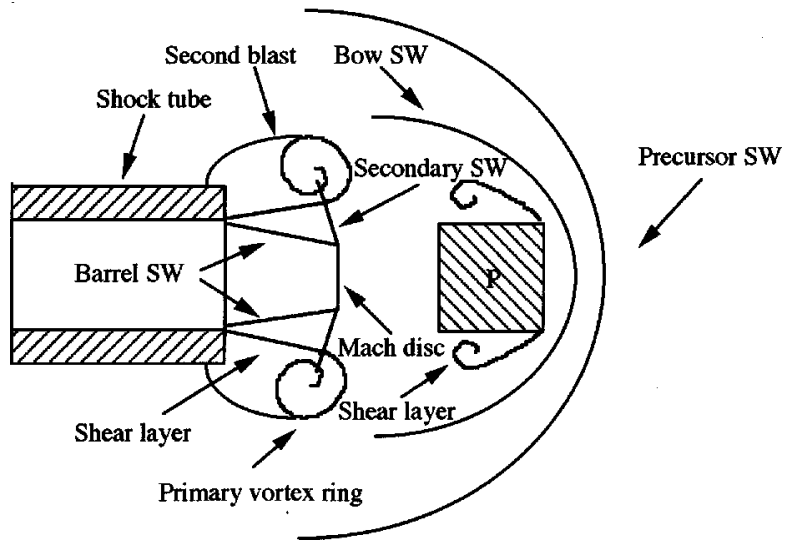

FIG. 1. Schematic of wave dynamic processes occurring in muzzle blast flows.

particularly is devoted to examining behaviors of the second blast wave when friction is present between the projectile and the shock tube wall. The friction in real cases varies case by case and it is approximated by assuming uniform distribution on the contact surface between the projectile and the shock tube wall. The dispersion controlled-scheme proposed by Jiang ${ }^{9}$ was used for solving the Euler equations assuming axisymmetric flows. Moving boundary conditions were applied to simulate the flying body. Three test cases were conducted by varying the friction intenseness, which is represented by the ratio of the pressure behind the projectile to that in front of it. The resulting wave dynamic processes observed from the numerical simulations are presented in a time sequence, and interpreted with the emphasis on motion of the second blast, affected by the friction, and its interaction with the projectile.

\section{GOVERNING EQUATIONS AND NUMERICAL METHODS}

Assuming that effects of viscosity and chemical reaction on wave dynamic processes in the present study are negligible, a hyperbolic system of the conservation laws for a perfect gas in axisymmetric coordinates can be written as

$$
\frac{\partial \mathbf{U}}{\partial t}+\frac{\partial \mathbf{F}}{\partial x}+\frac{\partial \mathbf{G}}{\partial r}+\frac{1}{r} \mathbf{S}=0
$$

where $\mathbf{U}, \mathbf{F}, \mathbf{G}$, and $\mathbf{S}$ denote the state variable, fluxes, and source, respectively, given by

$$
\begin{aligned}
& \mathbf{U}=\left(\begin{array}{c}
\rho \\
\rho u \\
\rho v \\
e
\end{array}\right), \quad \mathbf{F}=\left(\begin{array}{c}
\rho u \\
\rho u^{2}+p \\
\rho u v \\
(e+p) u
\end{array}\right), \\
& \mathbf{G}=\left(\begin{array}{c}
\rho v \\
\rho v u \\
\rho v^{2}+p \\
(e+p) v
\end{array}\right), \quad \mathbf{S}=\left(\begin{array}{c}
\rho v \\
\rho v u \\
\rho v^{2} \\
(e+p) v
\end{array}\right),
\end{aligned}
$$

where primitive variables in the unknown $\mathbf{U}$ are density $\rho$, velocity components $u$ and $v$, respectively. $p$ is the fluid

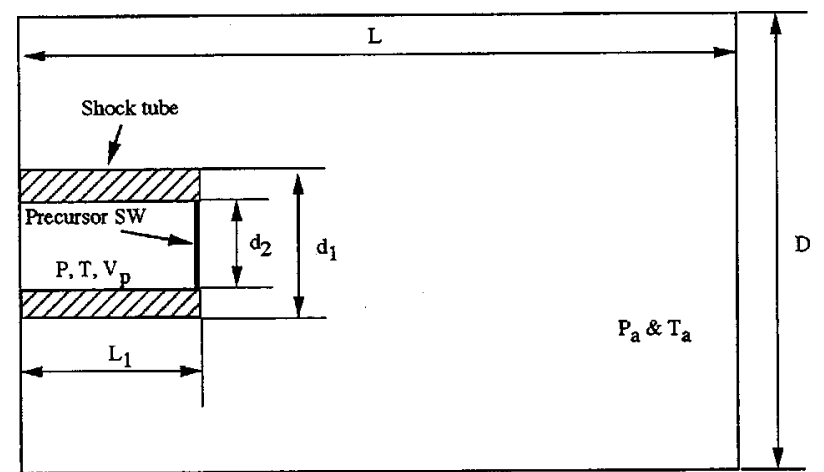

FIG. 2. Computational domain and dimension definitions for numerical simulations.

pressure and the total energy per unit volume $e$ related to the equation of state for the perfect gas is given by

$$
e=\frac{p}{\gamma-1}+\frac{\rho}{2}\left(u^{2}+v^{2}\right)
$$

where $\gamma$, the specific heat ratio, is taken as 1.4 in air.

The difference equations of Eq. (1) discretized in space using the dispersion-controlled scheme ${ }^{10}$ are given in the form of half discretion as

$$
\begin{aligned}
\left(\frac{\partial \mathbf{U}}{\partial t}\right)_{i, j}^{n}= & -\frac{1}{\Delta x}\left(\mathbf{H}_{i+1 / 2, j}^{n}-\mathbf{H}_{i-1 / 2, j}^{n}\right) \\
& -\frac{1}{\Delta r}\left(\mathbf{P}_{i, j+1 / 2}^{n}-\mathbf{P}_{i, j-1 / 2}^{n}\right)-\mathbf{S}_{i, j}^{n}
\end{aligned}
$$

with

$$
\begin{aligned}
& \mathbf{H}_{i+1 / 2, j}^{n}=\mathbf{F}_{i+(1 / 2) L, j}^{+}+\mathbf{F}_{i+(1 / 2) R, j}^{-}, \\
& \mathbf{P}_{i, j+1 / 2}^{n}=\mathbf{G}_{i, j+(1 / 2) L}^{+}+\mathbf{G}_{i, j+(1 / 2) R}^{-},
\end{aligned}
$$

where

$\mathbf{F}_{i+(1 / 2) L, j}^{+}=\mathbf{F}_{i, j}^{+}+\frac{1}{2} \boldsymbol{\Phi}_{\mathbf{A}}^{+} \operatorname{minimod}\left(\Delta \mathbf{F}_{i-1 / 2, j}^{+}, \Delta \mathbf{F}_{i+1 / 2, j}^{+}\right)$,

$\mathbf{F}_{i+(1 / 2) R, j}^{-}=\mathbf{F}_{i+1, j}^{-}-\frac{1}{2} \boldsymbol{\Phi}_{\mathbf{A}}^{-} \operatorname{minimod}\left(\Delta \mathbf{F}_{i+1 / 2, j}^{-}, \Delta \mathbf{F}_{i+3 / 2, j}^{-}\right)$,

$\mathbf{G}_{i, j+(1 / 2) L}^{+}=\mathbf{G}_{i, j, k}^{+}+\frac{1}{2} \boldsymbol{\Phi}_{\mathbf{B}}^{+} \operatorname{minimod}\left(\Delta \mathbf{G}_{i, j-1 / 2}^{+}, \Delta \mathbf{G}_{i, j+1 / 2}^{+}\right)$,

$\mathbf{G}_{i, j+(1 / 2) R}^{-}=\mathbf{G}_{i, j+1}^{-}-\frac{1}{2} \boldsymbol{\Phi}_{\mathbf{B}}^{-} \operatorname{minimod}\left(\Delta \mathbf{G}_{i, j+1 / 2}^{-}, \Delta \mathbf{G}_{i, j+3 / 2}^{-}\right)$,

$\Delta \mathbf{F}_{i+1 / 2, j}^{ \pm}=\mathbf{F}_{i+1, j}^{ \pm}-\mathbf{F}_{i, j}^{ \pm}, \quad \Delta \mathbf{G}_{i, j+1 / 2}^{ \pm}=\mathbf{G}_{i, j+1}^{ \pm}-\mathbf{G}_{i, j}^{ \pm}$,

$\mathbf{F}^{ \pm}=\mathbf{A}^{ \pm} \mathbf{U}, \quad \mathbf{G}^{ \pm}=\mathbf{B}^{ \pm} \mathbf{U}$,

and

$$
\boldsymbol{\Phi}_{A}^{ \pm}=\mathbf{I} \pm \beta \Lambda_{A}^{ \pm}, \quad \boldsymbol{\Phi}_{B}^{ \pm}=\mathbf{I} \pm \beta \Lambda_{B}^{ \pm},
$$

where $\mathbf{A}$ and $\mathbf{B}$ are the Jacobian matrices of $\partial \mathbf{F} / \partial \mathbf{U}$ and $\partial \mathbf{G} /$ $\partial \mathbf{U}$, respectively. I is a unit matrix, $\beta=\Delta t / \Delta x$, and $\Lambda_{A}$ and $\Lambda_{B}$ are vectors that consist of the eigenvalues of matrix $\mathbf{A}$ and $\mathbf{B}$, respectively. In these equations, the $(\cdot)^{+}$or $(\cdot)^{-}$ superscript denotes flux vector splitting according to Steger and Warming. ${ }^{10}$ Numerical solutions were marched in time by using the Runge-Kutta method of second-order accuracy. 


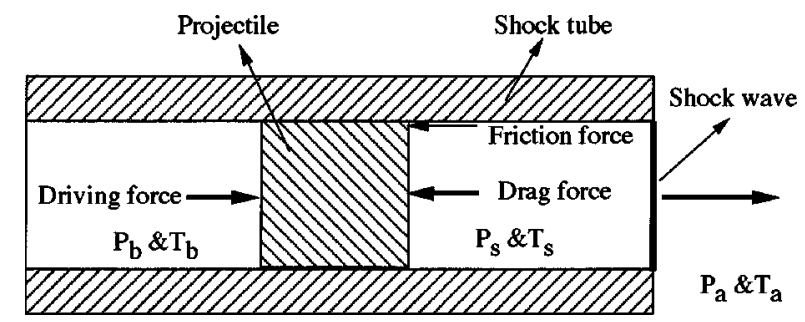

FIG. 3. Schematic diagram for initial condition descriptions and the friction definition.

The computational domain is shown in Fig. 2 with geometric sizes. The geometric parameters are: $L=600 \mathrm{~mm}$, $L_{1}=50 \mathrm{~mm}, d_{1}=30 \mathrm{~mm}, d_{2}=25 \mathrm{~mm}$, and $D=200 \mathrm{~mm}$. The inner diameter of the shock tube is accepted as a reference length and 50 mesh points are distributed along the radius for all the cases. The projectile is a 25 -mm-diam cylinder being $25 \mathrm{~mm}$ long and mass of $50 \mathrm{~g}$. The computation was carried out in the half of the computational domain because of symmetry of the flow field. Reflecting boundary conditions were specified both on solid walls and the axis of symmetry. Nonreflecting boundary conditions were applied at inflow and outflow boundaries. The equally spaced grid system of 3000 $\times 1000$ mesh points was used, and $250 \times 150$ points were distributed inside the shock tube. The CFL number accepted in all the computations is 0.5 and time steps are specified according to the required time for the flow state of interest.

In order to simulate a moving projectile, two grid systems are used in the present numerical simulation: the laboratory-fixed coordinate system (the fixed main mesh) and the projectile-fixed one (the moving mesh). The moving mesh containing the projectile moves on the fixed main mesh with the projectile speed. The surfaces of the projectile are traced step by step so that the moving boundary conditions consistent with the Euler equations and the numerical scheme could be applied on the surfaces. The detailed descriptions of the moving boundary conditions can be found in the reference by Jiang et al. ${ }^{8}$

\section{DESCRIPTION OF INITIAL CONDITIONS}

Oswatitsch ${ }^{11}$ pointed out that for high-speed projectiles, the most significant accelerations occur near the breech leaving the projectile speed relatively constant over the most of the latter portion of its in-bore trajectory, the properties of the gas slug at the muzzle can be obtained by using the Rankine-Hugoniot relations under the assumption that the gas velocity is equal to the projectile launch speed. Schmit et $a .^{4}$ carried out their theoretical analysis based on the assumption and the obtained results agree well with experiments. Considering the pioneer research work, and the friction between the projectile and the shock tube wall, we simplify initial conditions for numerical simulations as follows. In the initial stage, with the projectile moving down the shock tube, the precursor shock wave is taken as having arrived at the exit of the shock tube and the projectile is located behind the precursor shock wave at a certain distance that is determined with the projectile release time. The surrounding condition outside of the shock tube is ambient air at
$P_{a}=1$ atm and $T_{a}=297 \mathrm{~K}$, as shown in Fig. 3. Behind the precursor shock wave, the column of gas on either side of the projectile and the projectile itself all move at the same velocity, the so-called post-shock velocity, $V_{p}$, determined according to a given Mach number $M_{p}=V_{p} / c$, where $c$ is referred to as the sound speed in ambient air. During numerical simulations, the projectile speed will subsequently vary according to Newton's laws of motion. Using the projectile speed (the post-shock velocity) and the ambient air condition, the initial flow state between the precursor shock wave and the projectile can be specified using standard adiabatic shock relations.

As to the initial flow state behind the projectile, as shown in Fig. 3, it is assumed that the friction force acting on the projectile is proportional to the projectile surface area in contact with the tube wall, and furthermore, the driving force acting on the projectile is just sufficient to overcome the friction and the drag force to keep the projectile move at a constant speed as long as the projectile is moving totally inside the shock tube. When the projectile begins to move out of the shock tube, the total friction force is assumed to decrease linearly since its surface being in contact with the tube wall is reducing linearly. The total friction force can be calculated according to the length ratio of the projectile, that is, the length of the projectile being inside the shock tube to its total length. If the maximum friction force is set as a given percentage of the drag force acting on the front face of the projectile, which results from the pressure generated between the precursor shock and the projectile, for instance, $50 \%$ of the drag force, the pressure behind the projectile, $P_{b}$, will be taken as $1.5 P_{s}$, where $P_{s}$ is the post-shock pressure ahead of the projectile. The real friction may vary case by case in experiments, but the assumption represents the fact that the bigger friction force will induce stronger second blasts. According to the assumption, the state behind the projectile, described with density and temperature, can be calculated from the pressure, $P_{b}$, by using the Poisson's adiabatic equation for a perfect gas providing that the gas behind the projectile is compressed adiabatically from the gas state in front of the projectile to the pressure, $P_{b}$.

The initial condition described above may not be an exact analogy to the projectile motion in a tube, but does represent the key feature of the problem, that is, the high pressure propellant gas that induces the second blast wave is closely related with the friction.

\section{RESULTS AND DISCUSSION}

In the following discussion, a number of major wave dynamic processes will be identified, the details of which depend on the ratio of the pressure ahead of the projectile to that behind it, which varies with the friction between the projectile and the shock tube wall. The first process of these is the second blast overtaking the projectile. This process includes the leading shock of the blast, contact surface instability, and the behavior of the second jet flow, which thus correspond to the wave processes usually obscured in experiments such as, for example, the results shown by Glass ${ }^{2}$ and Schmidt et al. ${ }^{3}$ The second process occurs later when the 


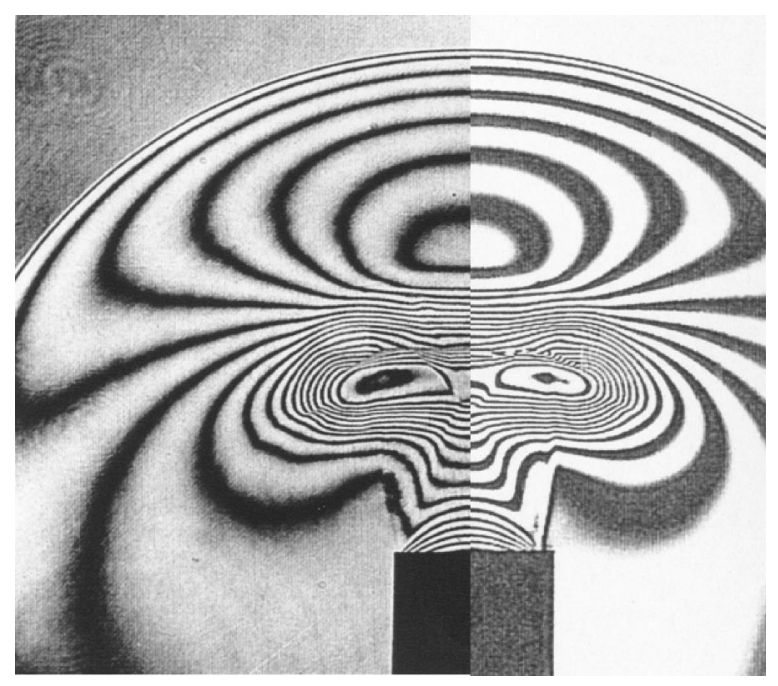

FIG. 4. Shock wave diffraction at the muzzle at a Mach number of 1.5.

second blast becomes weaker due to flow expansion; the projectile will overtake the blast again. During this process the bow shock is generated ahead of the projectile and interacts with the second blast first and with the first blast later. The third process being considered is the second blast itself, including its generation, motion, and interaction with the first blast. The last one is the acceleration of the projectile, which depends mainly on the pressure ratio set in the initial stage.

Three test cases are considered in this study, and achieved by setting the friction force at $25 \%, 50 \%$, and $75 \%$ of the drag force acting on the frontal surface of the projectile, respectively, so that the friction effect on the generation of the second blast, and its interaction with the projectile and the first blast, could be examined.

\section{A. Validation of numerical solutions}

For a numerical study on flow physics, the validation of numerical solutions has to be carried out to a certain extent. This is necessary because numerical simulations of fluid flows involve two essential steps: (1) selecting a suitable mathematical model that describes the physical phenomena of interest and (2) developing numerical techniques to compute a solution of the mathematical model using digital computers. Both steps generally introduce approximations in numerical simulations, therefore, the resulting numerical solutions may or may not represent the real fluid flows being considered. ${ }^{12}$ The validation of numerical simulations in this paper was reported by Jiang et al. (1999) ${ }^{8}$ and the main results are given here for completeness.

The muzzle blast flow is mainly dominated by two flow phenomena: one of them is the shock wave diffraction at the muzzle and other is the bow shock driven by the supersonic projectile. The validation strategy is chosen to check these two flow phenomena separately because suitable experimental results for the required validation are not available for the present study. Figure 4 shows the shock wave diffraction near the muzzle at a Mach number of 1.6, where an experimental interferogram is given in the left half, and a computational result is in right. It can be seen from the comparison

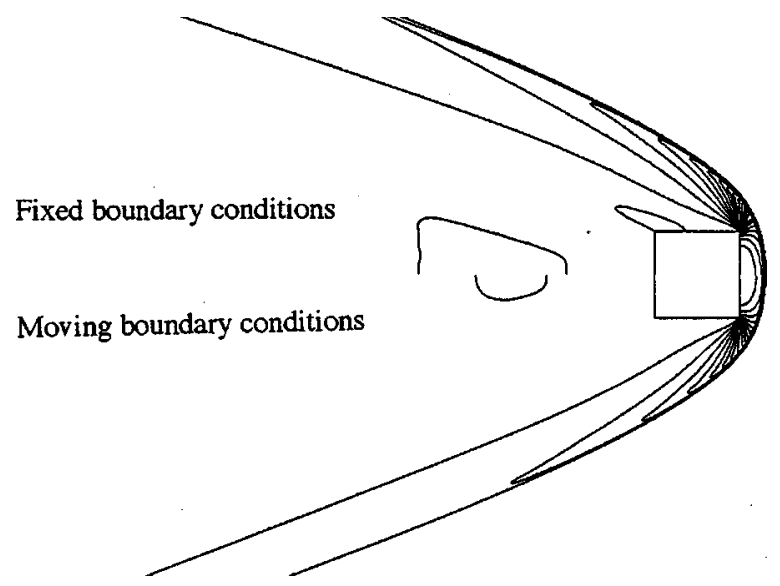

FIG. 5. Verification of moving boundary conditions.

that agreement between the computational result and the experimental data is excellent. This is not only because the number of fringes is identical but the distribution of the individual fringes matches well with each other with only minor exceptions. In fact, the largest deviation in fringe positions is less than half of the fringe interval. The results demonstrating verification of the moving boundary condition are presented in Fig. 5, where the numerical result calculated with moving boundary conditions in the projectile-fixed coordinates is presented in the lower half and the one in the laboratory-fixed coordinates is given in the upper half. Good agreement is also observable both from the stand-off distance of the bow shock, and from the number of isolines. The maximum discrepancy in the stagnation flow pressure is less than $7 \%$ of the free stream reference pressure, therefore, the accuracy of the moving boundary condition is regarded as being acceptable. The equally spaced grid system of 900 $\times 300$ mesh points was used for these validation tests.

\section{B. Wave processes in the case of $M_{p}=4.0$ and $P_{b} / P_{s}$ $=1.5$}

The first test case is carried out at the initial conditions of $M_{p}=4.0$ and $P_{b} / P_{s}=1.5$, where the friction is taken to be $50 \%$ of the drag force. A time sequence of its numerical results is given in Fig. 6, where isobars are plotted in the symmetrical lower half and isopycnics in the upper half. The arrangement enables shock waves to be distinguished from contact surfaces. The same display is also used in other figures in this paper and will be not mentioned again in the later discussion. For the projectile speed of $M_{p}=4.0$, the precursor shock wave driven down the shock tube propagates at $M_{s}=5.0$. Contour levels are scaled linearly between zero and the maximum value for each plot, and the number of contours is $\mathbf{5 0}$ for density and $\mathbf{1 0 0}$ for pressure to make interesting features stand out against the background. The maximum value obtained for this case is: $P / P_{a}=31.67$ for pressure, and $\rho / \rho_{a}=6.6788$ for density, where $P_{a}$ and $\rho_{a}$ denote the pressure and the density in ambient air.

Figure 6(a) shows a frame at $t=119.37 \mu \mathrm{s}$ after the precursor shock wave moves out of a shock tube. The high pressure gas behind the projectile expands out of the shock 

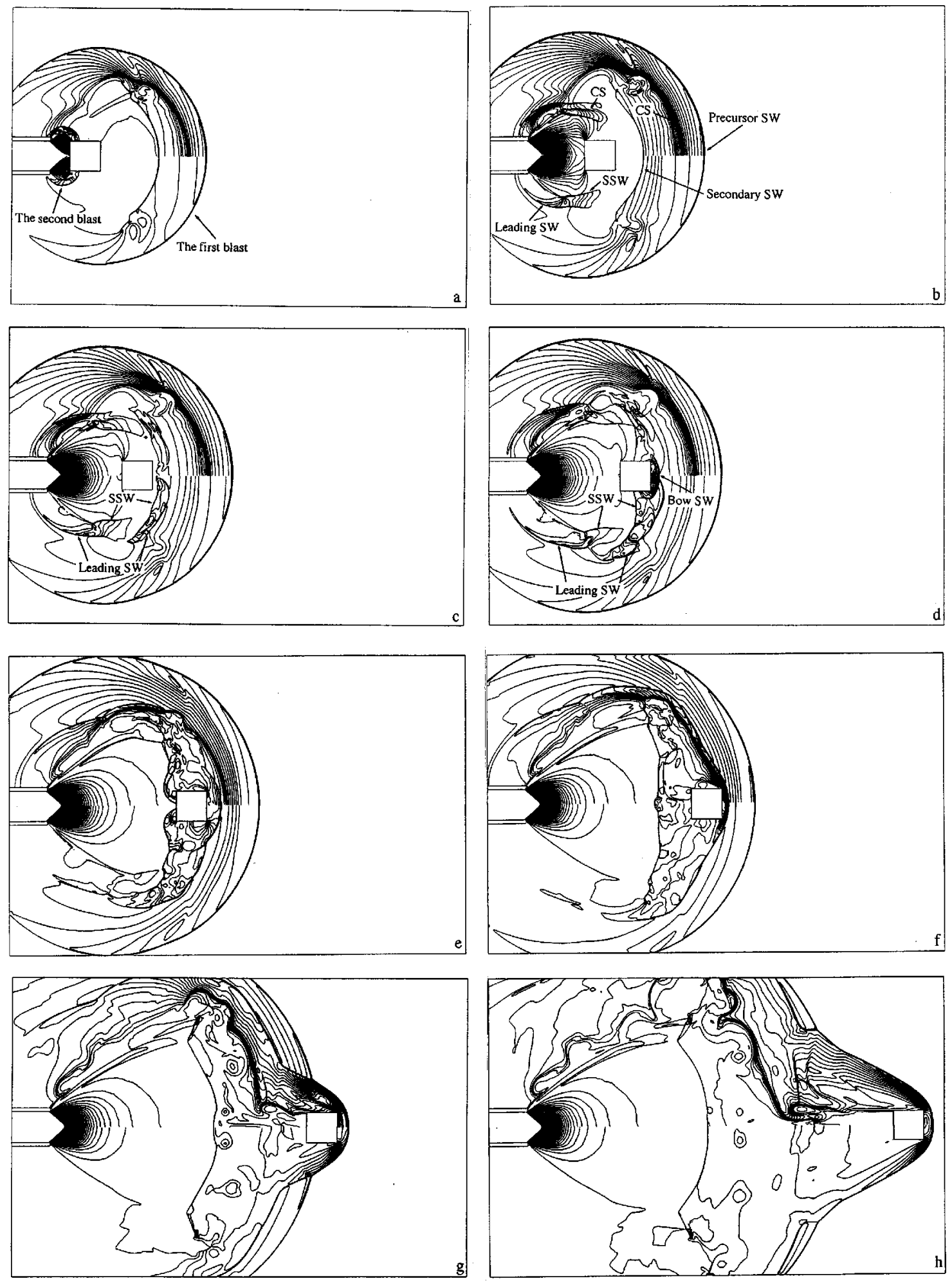

FIG. 6. Isopycnics (the upper half) and isobars (the lower half) of the muzzle blast at the projectile speed of $M_{p}=4.0$ and the pressure ratio of $P_{b}$ $=1.5 P_{s} . \mathrm{SW}$-shock wave; SSW—-second shock wave; leading SW-leading shock wave of the second blast.

tube and the second blast wave develops. The second blast consists of a leading shock wave, a contact surface, and an expansion fan. The wave system propagates outward, mainly in a radial direction but also along the side wall of the projectile. Meanwhile, the projectile moves inside the first jet where the particle velocity is almost equal to the projectile speed, therefore, there is nothing observable in front of the projectile. The precursor shock wave, the contact surface, and the secondary upward-facing shock in the first blast are approximately spherical in shape, and treated in more detail by Jiang et al. ${ }^{8}$

As the projectile continues to move downstream the axial part of the leading shock wave and the contact surface in the second blast disappear, as shown in Fig. 6(b) at $t$ 
$=141.07 \mu \mathrm{s}$. The reason for the apparent disappearance is that both the pressure and the density in the first jet behind the secondary shock wave are extremely low, almost close to zero, due to flow overexpansion. So, the overpressure generated due to the shock wave propagation within the region is so small that any shock wave could not be visible due to the small pressure and density differentials. As to the leading shock wave in the radial direction, it appears clearly, and follows by a contact surface and a secondary shock wave due to locally developed supersonic flows, as indicated in Fig. 6(b).

The frame at $t=151.97 \mu \mathrm{s}$ is shown in Fig. 6(c). As expected, the leading shock wave of the second blast reappears in front of the projectile, in which the density is higher, after it overtakes the secondary shock wave of the first blast. It is interesting to point out that the leading shock wave is traveling downstream and the secondary shock wave propagates upstream. By checking the pressure distribution along the axis of symmetry it is found that the downstream traveling wave rides on the upstream one when they meet. In the radial direction the leading shock wave is sweeping over the contact surface of the first blast at a varying angle, which will result in the Richtmyer-Meshkov instability that will be discussed later.

The projectile has penetrated through the upward-facing secondary shock wave at $t=162.77 \mu \mathrm{s}$, as shown in Fig. $6(d)$, thereby it is moving from a region with a gas velocity similar to the projectile speed to where the gas velocity is very much lower. This results in development of a bow shock wave ahead of the projectile because its speed is supersonic with respect to the gas ahead of it. As the projectile moves further downstream, the upward-facing secondary shock wave propagates backward relative to the projectile surface. Meanwhile, the leading shock wave of the second blast is imploding toward the axis of symmetry and interacts with the bow shock wave. It is also noted that the reappearance of this leading shock wave is not a continuous one, and actually is split by the primary vortex ring. This is a classic wave phenomenon discussed further in the research area of shock-wave/vortex interaction.

Diffraction of the secondary shock wave over the rear of the projectile is observed in Fig. 6(e) at $t=184.48 \mu \mathrm{s}$ and a contact surface follows. In front of the projectile, the bow shock wave overtakes the leading shock wave of the second blast and the resultant shock wave separates the second blast flow field from that disturbed only by the first blast. Figure 6(f) shows the impingement of the resultant shock wave on the contact surface of the first blast at $t=206.34 \mu \mathrm{s}$. This will result in development of the Richtmyer-Meshkov instability. Because of the curved shape of the shock wave, the interaction will make the contact surface develop easily into an unstable state.

The projectile overtaking the first blast is observed in Fig. $6(\mathrm{~g})$ at $t=260.45 \mu \mathrm{s}$, where the remainder of the blast behind the projectile is still observable. The RichtmyerMeshkov instability develops very rapidly from Figs. 6(f)6(h) at $t=325.59 \mu \mathrm{s}$. This implies that perturbations imposed by the second blast are stronger than those in case 3 presented by Jiang et al. ${ }^{8}$ The spherical upward-facing sec- ondary shock wave becomes well developed again in Fig. 6(h). The region bounded by the shock wave and the barrel shock wave appears longer and wider than the first blast because the second jet is more under-expanded.

\section{Interaction between the projectile and jet flows}

When the pressure ratio becomes higher, say the bigger friction exists between the projectile and the shock tube wall, not only do the projectile and the leading shock of the second blast overtake each other, but the projectile and the gas initially located behind it do so in a similar way. This can be observed in Fig. 7 where in each plot, velocity vectors are shown in the lower half and vorticity contours in the upper half in a time sequence. Figures $7(a)-7(d)$ show the results corresponding to Figs. 6(b)-6(e), respectively. From Fig. 7 (a), showing the frame at $t=130.22 \mu \mathrm{s}$, it is observed that the velocity vectors along the side wall of the projectile are longer than those ahead of the projectile, which indicates that the second jet created by the second blast is stronger than the first jet. This wave phenomenon can also be recognized from the distribution of vorticity, as shown in Fig. 7(b) at $t$ $=151.97 \mu \mathrm{s}$, where the two shear layers originating from two edges of the projectile develop in different directions: one toward the upstream and one downstream. It is because the higher gas pressure behind the projectile leads to a stronger flow expansion which results in a higher jet velocity, which results in the shear layer developing toward downstream. The gas behind the projectile overtakes the projectile in Fig. 7(b) and the projectile re-overtaking the gas is observed in Figs. 7(c) and 7(d) where the projectile moves out of the secondary shock wave at almost constant speed but the gas velocity decreases significantly after the gas passes though the secondary shock wave. The phenomenon is identical to that seen when a bullet emerges from the dusty propellant gases, as observed in many experimental pictures in which the process is not observable due to the propellant gas being full of smoke and dust.

\section{Bow shock wave generation}

The generation of the bow shock wave can be observed more clearly from schlieren photos which indicate the density gradient. A time sequence of numerical schlieren photos created with the numerical simulations of case 1 during bow shock wave generation is presented in Fig. 8. Figure 8(a) at $t=151.97 \mu \mathrm{s}$ shows that the projectile is moving in the first jet and going to approach the secondary shock wave or Mach disc. Because the difference between the projectile speed and the particle velocity in front of the projectile is minor, the projectile effect on the jet flow is not observable in front of the projectile. However, there is an observable wave phenomenon similar to shock wave diffraction, which results from the leading shock wave of the second blast diffracting over the frontal surface of the projectile, that is, the leading shock wave overtakes the projectile, because its speed is faster than the projectile. 

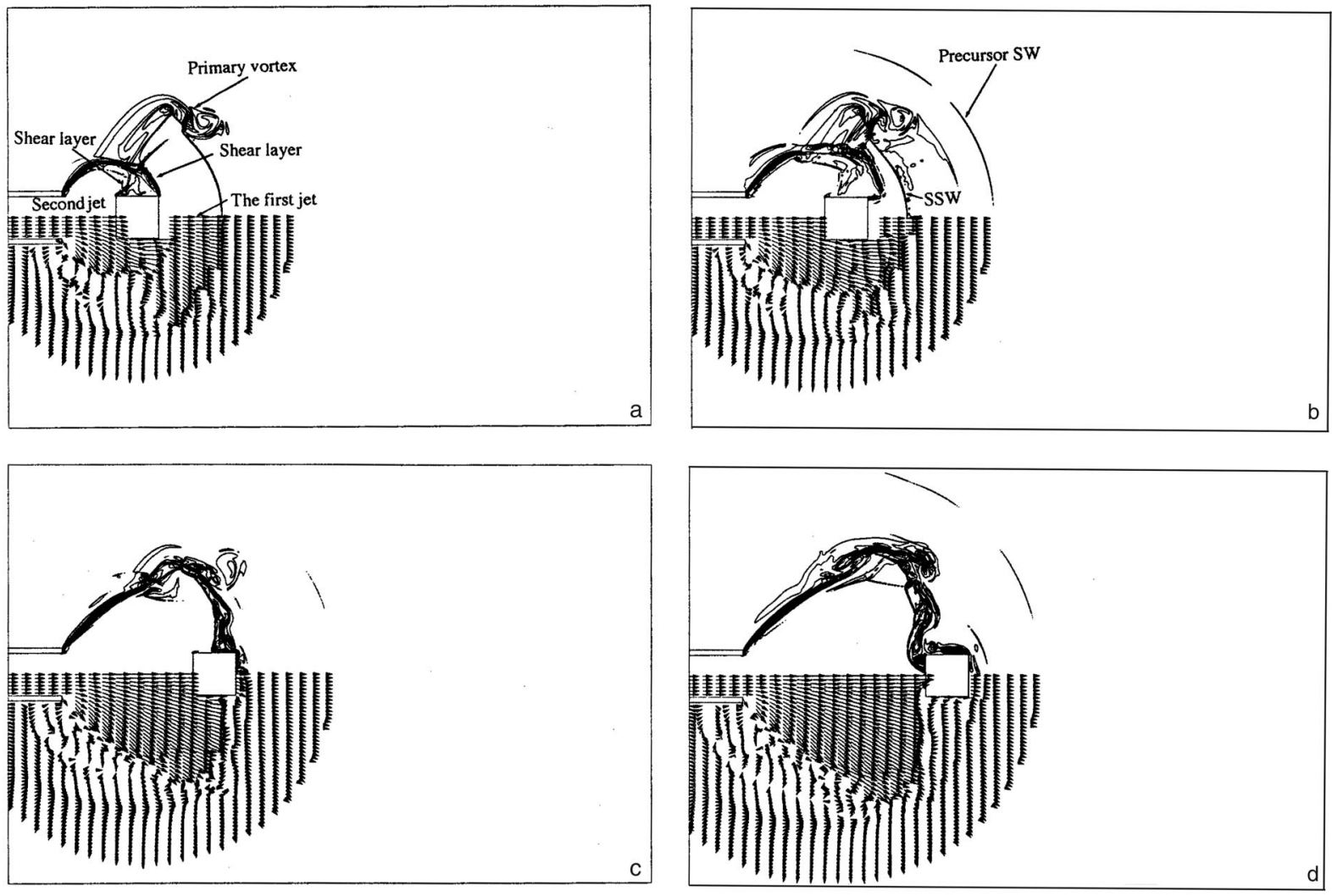

FIG. 7. Vorticity isolines (the upper half) and velocity vectors (the lower half) of the muzzle blast at the projectile speed of $M_{p}=4.0$ and the pressure ratio of $P_{b}=1.5 P_{s}$.

The second blast wave can be observed clearly in the flow field near the tube exit, where it propagates in the radial direction. From Figs. 8(a)-8(d), the leading shock wave sweeps over the contact surface induced by the first blast, which will lead to the Richtmyer-Meshkov instability that will be discussed further later. The leading shock wave joints itself in front of the projectile in Fig. 8(b) and develops into a noncircular wave front due to its propagation being on the background of the first blast, in which the particle velocity varies dramatically.

The bow shock wave occurs, first, in the corner of the projectile, as shown in Fig. 8(c) at $t=162.77 \mu$ s and then develops into shape in Fig. 8(d) at $t=173.12 \mu \mathrm{s}$, where it catches up with the leading shock wave of the second blast. Actually, the bow shock wave is much stronger than the second blast because the leading shock wave damps rapidly with the distance increase from the muzzle, but the bow shock wave is driven by the projectile at almost constant speed. The secondary shock wave diffracting over the rear surface of the projectile is observed in Fig. 8(d), which indicates that the projectile has moved out of the shock cell. It is important to point out that the bow shock wave is generated in the background flow created by the first blast and disturbed also by the second blast. Any asymmetric disturbance may lead to nonaxisymmetric bow shock wave generation, which may result in the projectile moving away from its original direction. This is an important problem in ballistic range.

\section{E. Interaction between moving shock waves}

The numerical schlieren photos created with the numerical results of case 1 at its later stages are presented in Fig. 9, and the interaction among moving shock waves and contact surfaces will be discussed in detail.

Since the frame shown in Fig. 9(a), the bow shock wave has caught up with the precursor shock wave, which leads to the so-called moving shock interaction. These waves are not only transient but also nonplanar. The interaction of these moving waves is observable in Fig. 9(b), where two contact surfaces appear. The one originating from the first triplepoint is created due to the interaction between the precursor shock wave and the bow shock wave. The other is generated due to the intensity difference of the bow shock wave before and after its interaction with the precursor shock wave. The phenomenon is indicated in Fig. 9(d). Considering a case where a shock wave propagates ahead and follows another shock wave, one could see that the post-shock pressure after the second shock wave catches up with the first one is less than the pressure induced by these two shock waves successively. This is because the stronger shock wave will induce higher enthalpy increase that results in bigger pressure loss. Such an interaction will lead to a series of expansions waves or a weak shock wave, and a contact surface. 


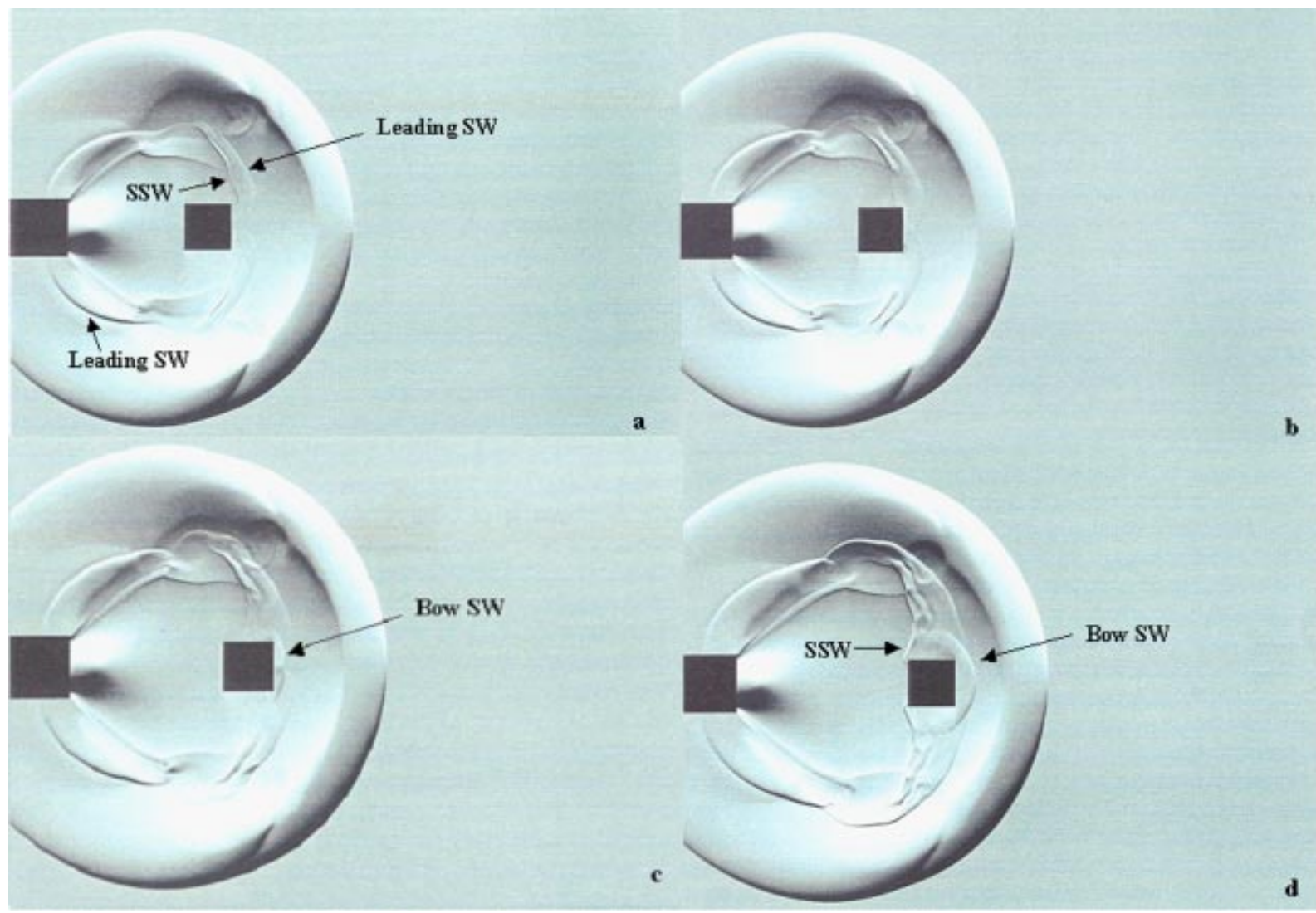

FIG. 8. A time sequence of numerical schlieren photos showing the bow shock wave generation in the muzzle blast at the projectile speed of $M_{p}=4.0$ and the pressure ratio of $P_{b}=1.5 P_{s}$.

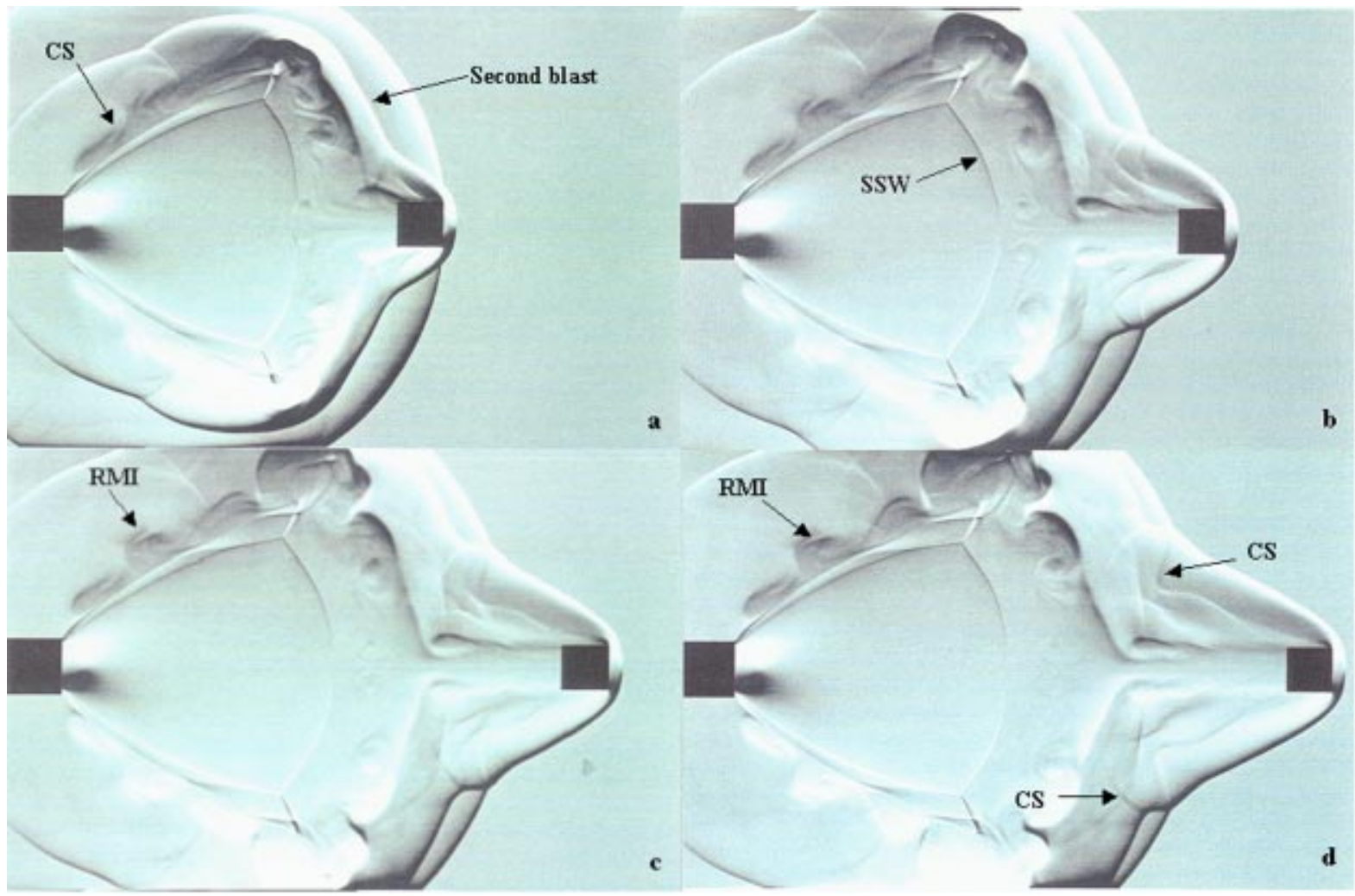

FIG. 9. A time sequence of numerical schlieren photos showing the moving shock wave interaction in the muzzle blast at the projectile speed of $M_{p}=4.0$ and the pressure ratio of $P_{b}=1.5 P_{s}$. CS—contact surface; RMI-Richtmyer-Meshkov instability. 

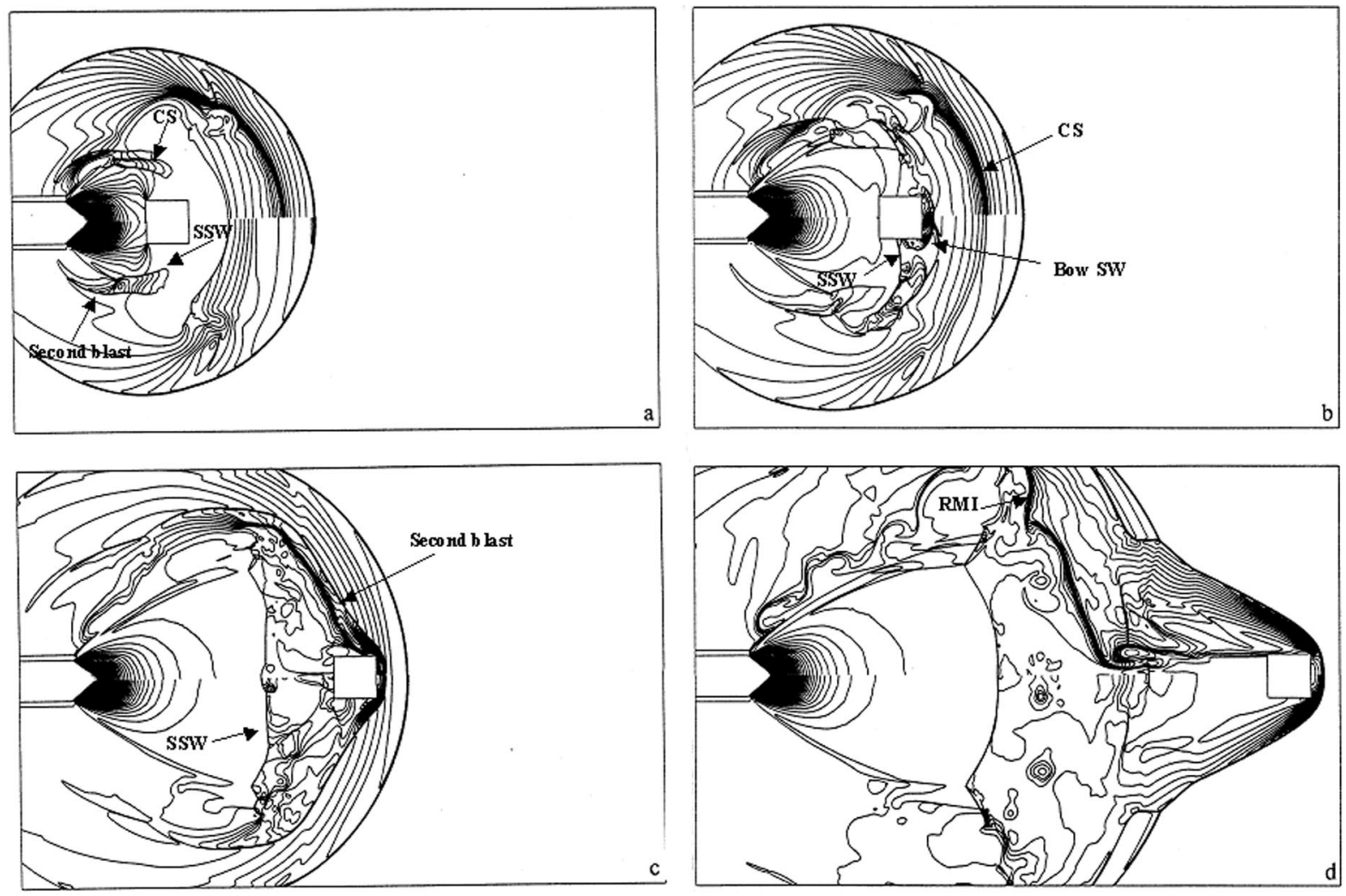

FIG. 10. Isopycnics (the upper half) and isobars (the lower half) of the muzzle blast at the projectile speed of $M_{p}=4.0$ and the pressure ratio of $P_{b}=1.25 P_{s}$.

The interaction of the leading shock wave with the contact surface has been mentioned in the above discussion when we observed the leading shock wave of the second blast sweeping over the contact surface at a varying angle. The resulting instability development is shown more clearly in Fig. 9. From Fig. 9(a), only small disturbances are observable on the contact surface. These disturbances develop rapidly from Figs. 9(b) -9 (c), and finally results in rolling up of the contact surface in Fig. 9(d) at $t=325.59 \mu$ s. Such instability was discussed in Ref. 8, but the contact surface appears more unstable in the present case. This is believed due to the stronger disturbances induced by the stronger leading shock wave resulting from the higher pressure ratio that represents the effect of friction.

\section{F. Role of the pressure ratio of $\boldsymbol{P}_{b} / \boldsymbol{P}_{s}$}

To explore the role of the pressure ratio in the blast generation, two other cases are conducted for comparison. The pressure ratio is set to be $P_{b}=1.25 P_{s}$ for case 2 and $P_{b}=1.75 P_{s}$ for case 3 . The initial condition indicates the total friction is $25 \%$ of the drag force in case 2 and $75 \%$ in case 3. Numerical results are given in Figs. 10 and 11, respectively, with a time sequence of isobars and isopycnics. Contour levels are the same as case 1 but the maximum values are $P / P_{a}=25.9$ and $\rho / \rho_{a}=5.68$ for case 2 , and $P / P_{a}=36.25$ and $\rho / \rho_{a}=7.45$ for case 3 .

Generally speaking, wave dynamic phenomena in these two cases appear similar to each other, for example, the gen- eration of the second blast in Figs. 10(a) and 11(a) at $t$ $=141.07 \mu \mathrm{s}$; the development of the bow shock wave in Figs. 10(b) and 11(b) at $t=162.77 \mu \mathrm{s}$; joining of the bow shock wave with the leading shock wave in Figs. 10(c) and 11 (c) at $t=206.34 \mu \mathrm{s}$; and the recovery of the spherical secondary shock wave, or the Mach disc, in the second jet flow in Figs. 10(d) and 11(d). However, there are some discrepancies resulting from the difference in the pressure ratio. The first of these is the interval between the leading shock wave and the contact surface of the first blast. It is narrower in Fig. 10(c), but wider in Fig. 11(c). This is because the second blast wave is stronger due to the higher gas pressure behind the projectile in case 3. So, the leading shock wave propagates faster and leaves the contact surface far behind. For the same reason, the leading shock wave catches up with the first blast front earlier in case 3, which can be identified by comparing Fig. 10(d) with Fig. 11(d) where the distance between these two blast fronts is shorter in case 3 . The second one is the development of the contact surface instability. The contact surface behind the precursor shock wave looks identical in Figs. 10(a) and 11(a), but it becomes more unstable in case 3 , which can be identified from the Richtmyer-Meshkov instability in Fig. 11(d). The last one is the size of the first shock cell in jet flows. The shock cell is bigger in Fig. 11(d), but smaller in Fig. 10(d).

In conclusion, the higher gas pressure behind the projectile produces a stronger second blast that leads to more intensive wave interaction, but the primary wave processes are not affected significantly. 

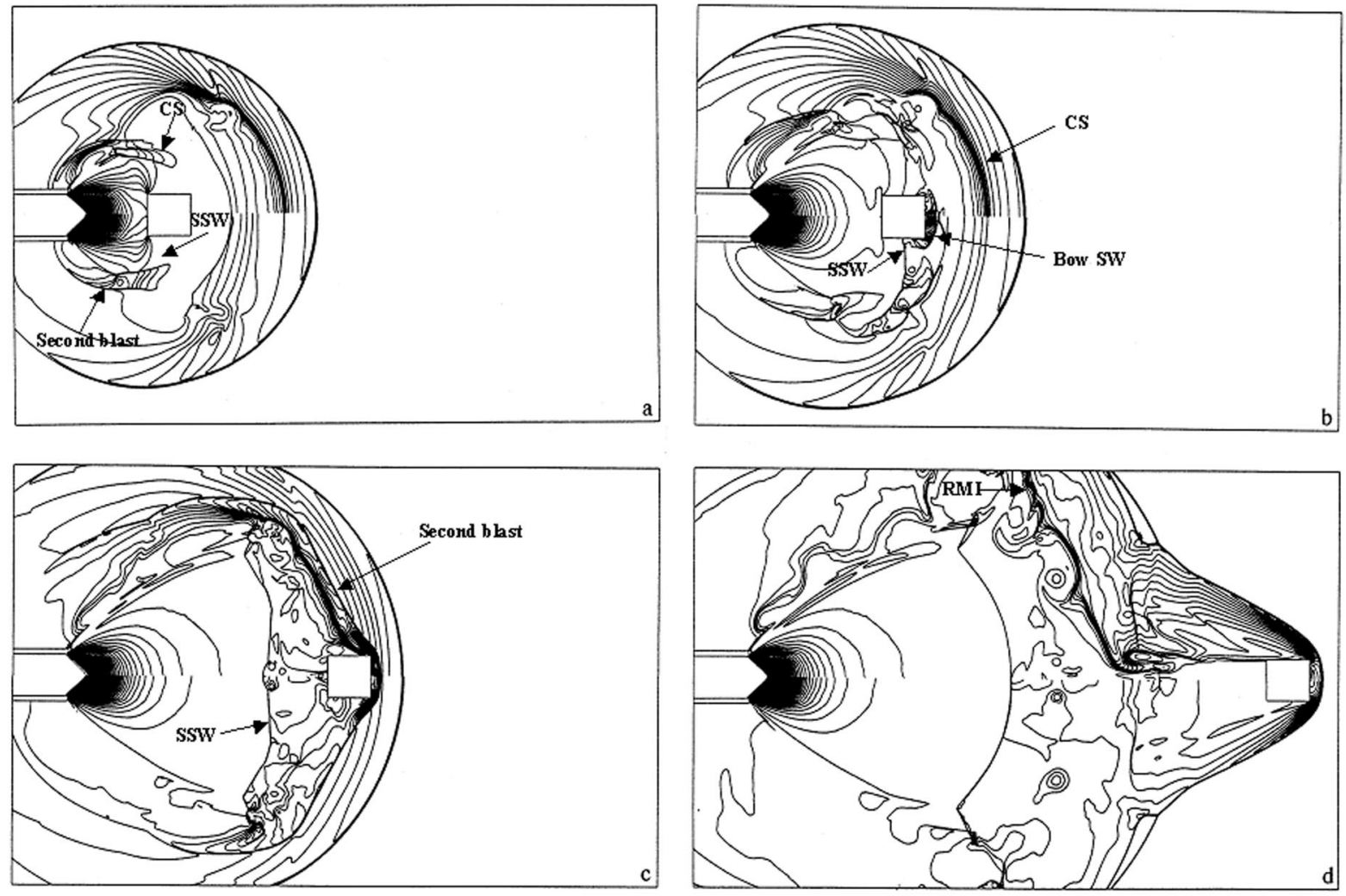

FIG. 11. Isopycnics (the upper half) and isobars (the lower half) at the projectile speed of $M_{p}=4.0$ and the pressure ratio of $P_{b}=1.75 P_{s}$.

\section{G. Acceleration and deceleration of the projectile}

It is apparent that varying the pressure in either side of the projectile will change the projectile speed and its acceleration process. To highlight the effect of the pressure change due to friction, the acceleration and deceleration histories of the projectile for cases 2 and 3 are presented in Figs. 12 and 13, respectively. From these figures it is observed that the acceleration and deceleration histories have the same tendency, but the maximum value of acceleration changes dramatically. The maximum value of acceleration in case 3 is about $45 \%$ higher than in case 2 , but the deceleration is almost the same level. There are also two additional features of interest to note. The first one is the identical acceleration distance in the two cases, which depends on the length of the

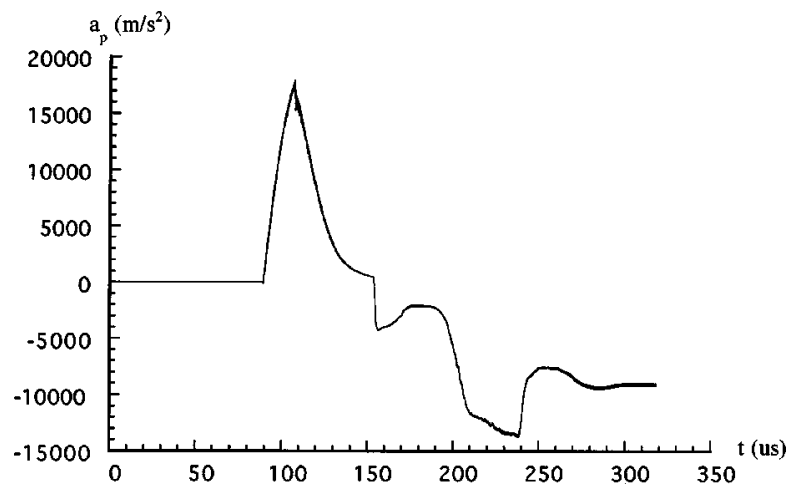

FIG. 12. Acceleration and deceleration of the projectile at the projectile speed of $M_{p}=4.0$ and the pressure ratio of $P_{b}=1.25 P_{s}$. first shock cell in the jet flow and is not affected by the second blast. Once the projectile catches up with the Mach disc of the shock cell, the drag force will increase sharply and the acceleration process will end. Although the acceleration magnitude is substantial, the actual change in the projectile speed is small due to the very short acceleration duration. The other is the similar deceleration process which depends on the projectile speed. After the projectile moves out of the shock cell, the drag force acting on the projectile is almost the same because the difference in the projectile speed and the flow velocity around it is minor in these two cases.

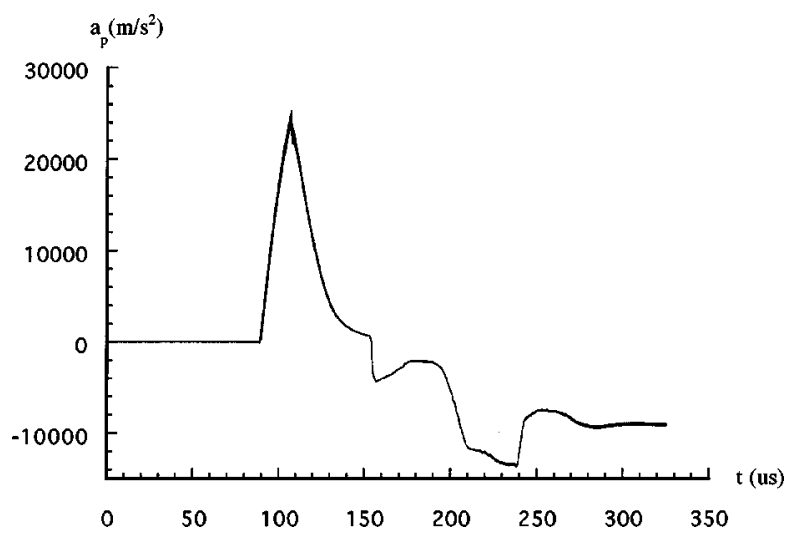

FIG. 13. Acceleration and deceleration of the projectile at the projectile speed of $M_{p}=4.0$ and the pressure ratio of $P_{b}=1.75 P_{s}$. 


\section{CONCLUSIONS}

Considering the friction between the projectile and the shock tube wall, the muzzle blast flow induced by a supersonic projectile was simulated by solving the Euler equations with a dispersion-controlled scheme implemented with moving boundary conditions. From numerical results, conclusions are draw as follows. When the pressure behind the projectile is higher than that in front of it, not only does the leading shock wave of the second blast overtake the projectile, but the gas behind the projectile does so, which results in more intensive shock-wave/moving-body interactions that lead to more complex wave dynamic processes. Moreover, the second blast catches up with the first one very quickly when the pressure ratio is higher, therefore, any theory that does not consider effects of the second blast on muzzle blast flows will fail to correctly predict the sonic boom related to the gun-firing noise problem. The higher pressure ratio does not affect wave dynamic processes significantly, but the acceleration of the projectile changes dramatically after it moves out of the shock tube. The effect of the pressure ratio is mainly limited to the near-field of muzzle blasts.

\section{ACKNOWLEDGMENTS}

The author would like to thank Professor B. Skew, University of the Witwatersrand, South Africa, and Professor K. Takayama, Tohoku University, Japan, for meaningful discus- sions. This work has been supported by the National Natural Science Foundation of China through Grant No. 90205027.

${ }^{1}$ W. B. Baker, Explosion in Air (University of Texas Press, Austin, 1974). ${ }^{2}$ I. I. Glass, Shock Waves and Man (The University of Toronto Press, Toronto, 1974).

${ }^{3}$ E. Schmidt and D. Shear, "Optical measurements of muzzle blast," AIAA J. 13, 1086 (1975).

${ }^{4}$ J. I. Erdos and P. D. Del Guidice, "Calculation of muzzle blast flowfields," AIAA J. 13, 1048 (1975).

${ }^{5}$ A. Merlen and A. Dyment, "Similarity and asymptotic analysis for gunfiring aerodynamics," J. Fluid Mech. 225, 497 (1991).

${ }^{6}$ M. Henner, M. Giraud, J. F. Legender, and C. Berner, "CFD computations of steady and non-reactive flow around fin-guided projectiles," Proceedings of the Third International Workshop on RAM Accelerators, Sendai, Japan, 16-18 July 1997, edited by K. Takayama and A. Sasoh, pp. 215221.

${ }^{7}$ S. Nonaka, K. Takayama, and C. Park, "Measurement of shock stand-off distance for sphere in ballistic range," AIAA Pap. 97-0563 (1997).

${ }^{8}$ Z. Jiang, K. Takayama, and B. W. Skews, "Numerical study on blast flowfields induced by supersonic projectiles discharged from shock tubes," Phys. Fluids 10, 277 (1998).

${ }^{9}$ Z. Jiang, K. Takayama, and Y. S. Chen, "Dispersion conditions for nonoscillatory shock capturing schemes and its applications," Computational Fluid Dyn. J. 2, 137 (1995).

${ }^{10}$ J. F. Steger and R. F. Warming, "Flux vector splitting of the inviscid gasdynamic equations with applications to finite difference methods," J. Comput. Phys. 40, 263 (1981).

${ }^{11}$ K. Oswatitsch, "Intermediate ballistics," DVL Report No. 358, June 1964, Deutschen Versuchsanstalt fur Luft- und Raumfahrt, Aachen, Germany.

${ }^{12} \mathrm{Z}$. Jiang and K. Takayama, "An investigation into the validation of numerical solutions of complex flowfields," J. Comput. Phys. 151, 479 (1999). 